\title{
Suicide among agricultural, forestry and fishery workers: a systematic literature review and meta-analysis
}

\author{
J. Klingelschmid, A. Milner, I. Khireddine-Medouni, K. Witt, E.C. Alexopoulos, S. Toivanen, A.D. LaMontagne, \\ J-F. Chastang, I. Niedhammer \\ Scand J Work Environ Health 2018; 44 (1): 3-15
}

DOI: 10.12961/aprl.2018.21.02.8

\section{SUICIDIO ENTRE LOS TRABAJADORES AGRÍCOLAS, FORESTALES Y PESQUEROS: UNA REVISIÓN SISTEMÁTICA DE LA LITERATURA Y UN METAANÁLISIS.}

\section{RESUMEN}

Objetivos: Cuantificar el riesgo de suicidio entre los trabajadores agrícolas, forestales y pesqueros, y analizar las posibles variaciones del riesgo dentro de esta población.

Métodos: Revisión sistemática de la literatura y un metaanálisis de los años 1995 al 2016 utilizando MEDLINE y siguiendo las recomendaciones de PRISMA. Se calculó el efecto agrupado del riesgo de suicidio entre la población de interés mediante la técnica del metaanálisis. Se realizaron análisis de subgrupos para evaluar si el tamaño del efecto difería según la población o las características del estudio. La meta regresión se utilizó para identificar fuentes de heterogeneidad.

Resultados: La revisión sistemática identificó 65 estudios, de los cuales se incluyeron 32 en el metaanálisis. El tamaño del efecto agrupado fue de 1,48 (IC95\% 1,30-1,68). El análisis de subgrupos mostró que el efecto varió según el área geográfica, por ejemplo, en Japón mostró un riesgo más alto. Las siguientes características contribuyeron a la varianza entre los estudios: grupo de referencia, medida del tamaño del efecto y diseño del estudio.

Conclusiones: Los hallazgos sugieren un exceso de riesgo de suicidio entre los trabajadores agrícolas, forestales y pesqueros, y demostraron que este exceso puede ser incluso mayor para estos grupos de trabajadores en Japón. Esta revisión destaca la necesidad de implementar políticas de prevención del suicidio enfocado a esta población específica de trabajadores. También se necesita más investigación para comprender los factores fundamentales que pueden aumentar el riesgo de suicidio en este colectivo.

\section{COMENTARIO}

El suicidio es un tema de gran interés por su magnitud e impacto $^{1}$. Diariamente, se producen en el mundo de 8.000 a 10.000 intentos de suicidio, de los que 1.000 acaban en muerte. Las estimaciones de la Organización Mundial de la Salud (OMS) señalan el suicidio como la décima causa de muerte con una tasa 14,5 muertes por cada 100.000 personas por año ${ }^{2}$.

Las tasas de suicidio varían según diferentes factores entre los que se incluye la ocupación; por ejemplo, en Colorado (Estados Unidos), los trabajadores del sector primario tuvieron la tasa ajustada por edad de suicidio más elevada $(475,6$ por 100.000 habitantes) durante el periodo 2004-2006, en comparación con otros sectores ${ }^{3}$.

En los últimos años, ha habido una serie de investigaciones que examinan la relación entre la ocupación y el suicidio. Una revisión sistemática y metaanálisis ${ }^{4}$ publicada en 2013 con 34 estudios incluidos, encontró que los agricultores y los pescadores tenían un riesgo de suicidio significativamente más elevado que otra población ocupada. A pesar de que en esta revisión sólo se incluyeron 9 estudios en trabajadores del sector primario, es la primera vez que se ofrece una visión general del suicidio de los trabajadores agrícolas, forestales y pesqueros.

El suicidio es un fenómeno social y multifactorial. La presente revisión encuentra un exceso de riesgo en los tres grupos estudiados. Entre las explicaciones que argumentan los autores se encuentran el acceso fácil a agentes letales como armas de fuego, monóxido de carbono, y sustancias tóxicas como plaguicidas ${ }^{5}$. La ingesta de plaguicidas es el método principal de suicidio, siendo responsable de alrededor del $30 \%{ }^{6}$. La agricultura es el sector profesional líder en el uso de plaguicidas en el mundo, con una facturación de aproximadamente 40.000 millones de dólares en el año $2010^{7}$.

El estrés laboral también es un factor bien relacionado con el suicidio de los agricultores. En concreto, las presiones financieras atribuibles a las fluctuaciones inesperadas del mercado, la preocupación por la política agrícola y las condiciones climáticas impredecibles, son fuentes de estrés reconocidas por los agricultores $^{8}$. Por lo tanto, posiblemente estos factores puedan aplicarse no solo a los agricultores, sino también a los trabajadores forestales y pesqueros.

Sección coordinada por:

Consol Serra (consol.serra@upf.edu).

Ma.del Mar Seguí (mm.segui@ua.es). 
La mayoría de agricultores, trabajadores forestales y pesqueros, trabajan al aire libre. Esta exposición laboral a condiciones climáticas adversas (exceso o falta de luz solar, cambios de temperatura) también se ha asociado al suicidio. Así, por ejemplo, se observa una estacionalidad asociada con una mayor tasa de suicidio. En Finlandia, los trabajadores forestales mostraron una tasa alta de suicidio en invierno, comparando con los otros grupos ocupacionales 9 . La mayor parte de los trabajos en bosques se realiza durante los meses de invierno, expuestos a oscuridad ambiental y al frío.

El entorno del trabajo del sector primario tiene un conjunto de riesgos potencialmente peligrosos para la salud mental relacionados con el aislamiento social y la falta de apoyo social ${ }^{10}$, porque estos grupos de personas normalmente viven en zonas más despobladas y con menos posibilidad de socialización. Según las propias condiciones de trabajo, como trabajan lejos de la ciudad, los agricultores también tienen niveles de acceso deficiente a los servicios de atención médica, como en Australia ${ }^{11}$.

Esta revisión incluye solo estudios realizados en países de altos ingresos como Europa, Norte América, Australia, Nueva Zelanda y Japón, mostrando claras diferencias por zonas geográficas. Sobre todo, destaca que los trabajadores agrícolas, forestales y pesqueros en Japón tienen un riesgo de suicidio más elevado (efecto agrupado 3,07, IC 95\% 2,37-3,98) que en otros países.

En España, el sector agrario agrupa alrededor del 4\% de la población ocupada. Hasta la fecha no hay ninguna investigación sobre suicidio en trabajadores agrícolas, forestales y pesqueros. Es importante identificar cuáles son los factores que influyen en el suicidio de las ocupaciones en este grupo ocupacional para fortalecer las medidas preventivas para estas personas.

\section{Guanlan Zhao}

zhaoguanlan0810@gmail.com

Área de Medicina y Salud Pública. Universidad de Alicante.

Alicante. España.

\section{REFERENCIAS}

1. Hawton K, Heeringen K van. Suicide. The Lancet. 2009; 373: 1372-81.

2. WHO | Download the World report on violence and health [Internet]. WHO. [cited 01 Mar 2018]. Available from: http://www.who.int/violence_injury_prevention/violence/world_report/chapters/en/.

3. Stallones L, Doenges T, Dik BJ, Valley MA. Occupation and suicide: Colorado, 2004-2006. Am J Ind Med. 2013; 56: 1290-5.

4. Milner A, Spittal MJ, Pirkis J, LaMontagne AD. Suicide by occupation: systematic review and meta-analysis. Br J Psychiatry. 2013; 203: 409-16.

5. Milner A, Witt K, Maheen H, LaMontagne AD. Access to means of suicide, occupation and the risk of suicide: a national study over 12 years of coronial data. BMC Psychiatry. 2017; 17: 125.

6. Gunnell D, Eddleston M, Phillips MR, Konradsen F. The global distribution of fatal pesticide self-poisoning: systematic review. BMC Public Health. 2007; 7: 357. 7. Levêque-Morlais N, Tual S, Clin B, Adjemian A, Baldi I, Lebailly P. The AGRIculture and CANcer (AGRICAN) cohort study: enrollment and causes of death for the 2005-2009 period. Int Arch Occup Environ Health. 2015;88:61-73.

8. Nishimura M, Terao T, Soeda S, Nakamura J, Iwata N, Sakamoto K. Suicide and occupation: further supportive evidence for their relevance. Prog Neuropsychopharmacol Biol Psychiatry. 2004; 28: 83-7.

9. Koskinen O, Pukkila K, Hakko H, Tiihonen J, Väisänen E, Särkioja T, et al. Is occupation relevant in suicide? J Affect Disord. 2002; 70: 197-203.

10. Hossain D, Eley R, Coutts J, Gorman D. Mental health of farmers in Southern Queensland: issues and support. Aust J Rural Health. 2008; 16: 343-8. 11. Fragar L, Depczynski J, Lower T. Mortality patterns of Australian male farmers and farm managers. Australian Journal of Rural Health. 2011; 19: 179-84. 